\title{
ST-segment elevation myocardial infarction in post-COVID-19 patients: A case series
}

\author{
Shiun Woei Wong ${ }^{1,3}{ }_{M R C P}$, Bingwen Eugene Fan ${ }^{2,3}{ }_{M R C P}$, Wenjie $\underline{\text { Huang }}{ }^{1,3}{ }_{\text {MRCP }}$, Yew Woon $\underline{\text { Chia }}{ }^{1,3}{ }_{\text {FRCPEd }}$
}

\begin{abstract}
Coronavirus disease 2019 (COVID-19) is associated with an increased risk of thromboembolic events in the acute setting. However, the abnormal thrombotic diathesis is not known to persist into the recovery phase of COVID-19 infection.

We described 3 cases of ST-segment elevation myocardial infarction in healthy male patients who recovered from COVID-19 with no prior cardiovascular risk factors. They shared features of elevated von Willebrand factor antigen, factor VIII and D-dimer level. One patient had a borderline positive lupus anticoagulant. Intravascular ultrasound of culprit vessels revealed predominantly fibrotic plaque with minimal necrotic core. Clot waveform analysis showed parameters of hypercoagulability. They were treated with dual antiplatelet therapy, angiotensin-converting-enzyme inhibitor, beta blocker and statin. These cases highlight the strong thrombogenic nature of COVID-19 that persisted among patients who recovered from infection. Several suspected mechanisms could explain the association between vascular thrombosis in the convalescent period (endothelial dysfunction, hypercoagulability, systemic inflammatory response and vasculopathy). Additional studies on "long COVID" are essential for identifying endotheliopathy and thrombotic sequalae.
\end{abstract}

Ann Acad Med Singap 2021;50:425-30

Keywords: Coronary artery disease, hypercoagulability, pandemic, thrombosis

Coronavirus disease 2019 (COVID-19) has been associated with thromboembolic phenomenon in the early phase of disease. Growing evidence suggests a hypercoagulable state as well as abnormal platelet activation, impaired fibrinolysis, and endothelial dysfunction in COVID-19 patients, resulting in thrombosis. ${ }^{1-3}$ The lungs are thought to be the epicentre of thrombosis, where thrombosis may manifest as in situ pulmonary thrombosis, as well as systemic microand macrovascular thrombosis. Little is known about post-COVID-19 thrombotic complications. ${ }^{4}$

Singapore adopted a mass screening strategy for its foreign workers after COVID-19 clusters were discovered in their dormitories. ${ }^{5}$ We report 3 cases of healthy male foreign workers who presented with massive acute myocardial infarction (AMI) after recovery from COVID-19. All 3 had raised immunoglobulin G levels for severe acute respiratory syndrome coronavirus-2 (SARS-CoV-2) that is indicative of seroconversion
(Table 1). Prior to their AMI presentation, the cases were asymptomatic and belonged to a low-risk group. They did not require hospital admission nor thromboprophylaxis and were quarantined at isolation facilities. $^{6}$

Case 1. A 38-year-old man, with no significant cardiovascular risk factors, was admitted to the emergency department with an out-of-hospital ventricular fibrillation (VF) cardiac arrest. He presented 80 days after his positive SARS-CoV-2 antibody serology on 9 July 2020 . He was successfully resuscitated, and a subsequent electrocardiogram (ECG) showed STsegment elevation in anterior leads. He was intubated for airway protection and started on vasopressor support for cardiogenic shock. Urgent coronary angiogram showed single vessel coronary artery disease with a complete occlusion of the left anterior descending artery (LAD) (Fig. 1A). Intravascular ultrasound (IVUS) with Eagle Eye IVUS Catheter (Philips Healthcare,

\footnotetext{
${ }^{1}$ Department of Cardiology, Tan Tock Seng Hospital, Singapore

${ }^{2}$ Department of Haematology, Tan Tock Seng Hospital, Singapore

${ }^{3}$ Lee Kong Chian School of Medicine, Nanyang Technological University, Singapore

Correspondence: Dr Shiun Woei Wong, Department of Cardiology, Tan Tock Seng Hospital, 11 Jalan Tan Tock Seng, Singapore 308433.

Email: shiun_woei_wong@ttsh.com.sg
} 
Table 1. Characteristics and investigation of 3 cases of post-COVID-19 acute myocardial infarction

\begin{tabular}{|c|c|c|c|}
\hline & Case 1 & Case 2 & Case 3 \\
\hline Age, years & 38 & 50 & 36 \\
\hline Sex & Male & Male & Male \\
\hline Cardiovascular risk factors & None & None & None \\
\hline SARS-CoV-2 RT-PCR results and dates & $\begin{array}{l}\text { Negative } \\
\text { 24 Jun, } 27 \text { Sep, } \\
\text { 28 Sep, } 3 \text { Oct } 2020\end{array}$ & $\begin{array}{l}\text { Positive } \\
4 \text { Jun } 2020 \\
\text { Negative } \\
29 \text { Nov, } 30 \text { Nov } 2020\end{array}$ & $\begin{array}{l}\text { Negative } \\
6 \text { Jun, } 14 \text { Jul, } 30 \text { Nov } 2020\end{array}$ \\
\hline $\begin{array}{l}\text { SARS-CoV-2 total antibody result and } \\
\text { date }\end{array}$ & $\begin{array}{l}\text { Positive } \\
9 \text { Jul } 2020\end{array}$ & $\begin{array}{l}\text { Positive } \\
8 \text { Jun } 2020\end{array}$ & $\begin{array}{l}\text { Positive } \\
18 \text { Jun } 2020\end{array}$ \\
\hline \multirow[t]{2}{*}{ Arterial event } & 27 Sep 2020 & 29 Nov 2020 & 30 Nov 2020 \\
\hline & $\begin{array}{l}\text { VF arrest, myocardial } \\
\text { infarction, occlusion of LAD }\end{array}$ & $\begin{array}{l}\text { VF arrest, myocardial infarction, } \\
\text { occlusion of proximal LAD }\end{array}$ & $\begin{array}{l}\text { Myocardial infarction, occlusion } \\
\text { of LAD }\end{array}$ \\
\hline $\begin{array}{l}\text { No. of days from positive serology to } \\
\text { thrombosis }\end{array}$ & 80 & 174 & 165 \\
\hline $\begin{array}{l}\text { D-dimer, } \mu \mathrm{g} / \mathrm{mL} \\
\text { (reference range }<0.5 \mu \mathrm{g} / \mathrm{mL} \text { ) }\end{array}$ & $>4$ & 1.02 & 0.43 \\
\hline $\begin{array}{l}\text { Fibrinogen, } \mathrm{g} / \mathrm{L} \\
\text { (reference range } 1.8-4.5 \mathrm{~g} / \mathrm{L} \text { ) }\end{array}$ & 5.2 & 5.7 & 3.6 \\
\hline $\begin{array}{l}\text { Activated partial thromboplastin time, s } \\
\text { (reference range } 27-37 \mathrm{~s} \text { ) }\end{array}$ & 31.8 & 26.5 & 28.8 \\
\hline $\begin{array}{l}\text { Prothrombin time, } \mathrm{s} \\
\text { (reference range } 11-14 \mathrm{~s} \text { ) }\end{array}$ & 15.7 & 13.7 & 12.7 \\
\hline Lupus anticoagulant & Weakly present & Absent & Absent \\
\hline $\begin{array}{l}\text { VWF antigen, } \% \\
\text { (reference range } 56-160 \% \text { ) }\end{array}$ & 366 & 215 & 92 \\
\hline $\begin{array}{l}\text { Factor VIII, \% } \\
\text { (reference range 60-150\%) }\end{array}$ & 273 & 338 & 162 \\
\hline Treatment & $\begin{array}{l}\text { Aspirin, ticagrelor, } \\
\text { drug eluting stent } \\
\text { implantation }\end{array}$ & $\begin{array}{l}\text { Dual antiplatelet therapy, drug } \\
\text { eluting stent implantation, } \\
\text { glycoprotein IIb/IIIa inhibitor }\end{array}$ & $\begin{array}{l}\text { Dual antiplatelet therapy, } \\
\text { drug eluting stent implantation }\end{array}$ \\
\hline Follow-up and outcome & $\begin{array}{l}\text { Successful treatment, } \\
\text { undergoing rehabilitation }\end{array}$ & $\begin{array}{l}\text { Successful treatment, discharge } \\
\text { on day } 12\end{array}$ & $\begin{array}{l}\text { Successful treatment, } \\
\text { discharge on day } 6\end{array}$ \\
\hline
\end{tabular}

LAD: left anterior descending artery; RT-PCR: reverse transcription-polymerase chain reaction; VF: ventricular fibrillation; VWF: von Willebrand factor

Cambridge, US) revealed a minimal amount of clot, which was retrieved using a thrombectomy device. Virtual histology (via IVUS) showed a focal, heavily fibrous plaque burden of $59 \%$ with minimal necrotic core and minimal lumen diameter of $2.1 \mathrm{~mm}$ in the ostial LAD (Fig. 1B). A drug-eluting stent $(3.8 \times 40 \mathrm{~mm})$ was subsequently implanted in the LAD with optimal result and an intraaortic balloon catheter was inserted for mechanical circulatory support.

The patient was initiated on our protocolised post-cardiac arrest care bundle including targeted temperature management at 33 degrees Celsius in the cardiac intensive care unit. Chest X-ray revealed mild pulmonary congestion with no evidence of consolidation. His reverse transcription-polymerase chain reaction (RT-PCR) results for COVID-19 was negative. Transthoracic echocardiography (TTE) revealed moderate left ventricular systolic dysfunction (left ventricular ejection fraction $40 \%$ ) with hypokinesia in the LAD territory. Laboratory results showed elevated von Willebrand factor (VWF) antigen of $366 \%$, factor VIII $273 \%$, elevated D-dimer $2.98 \mu \mathrm{g} / \mathrm{mL}$, and borderline 


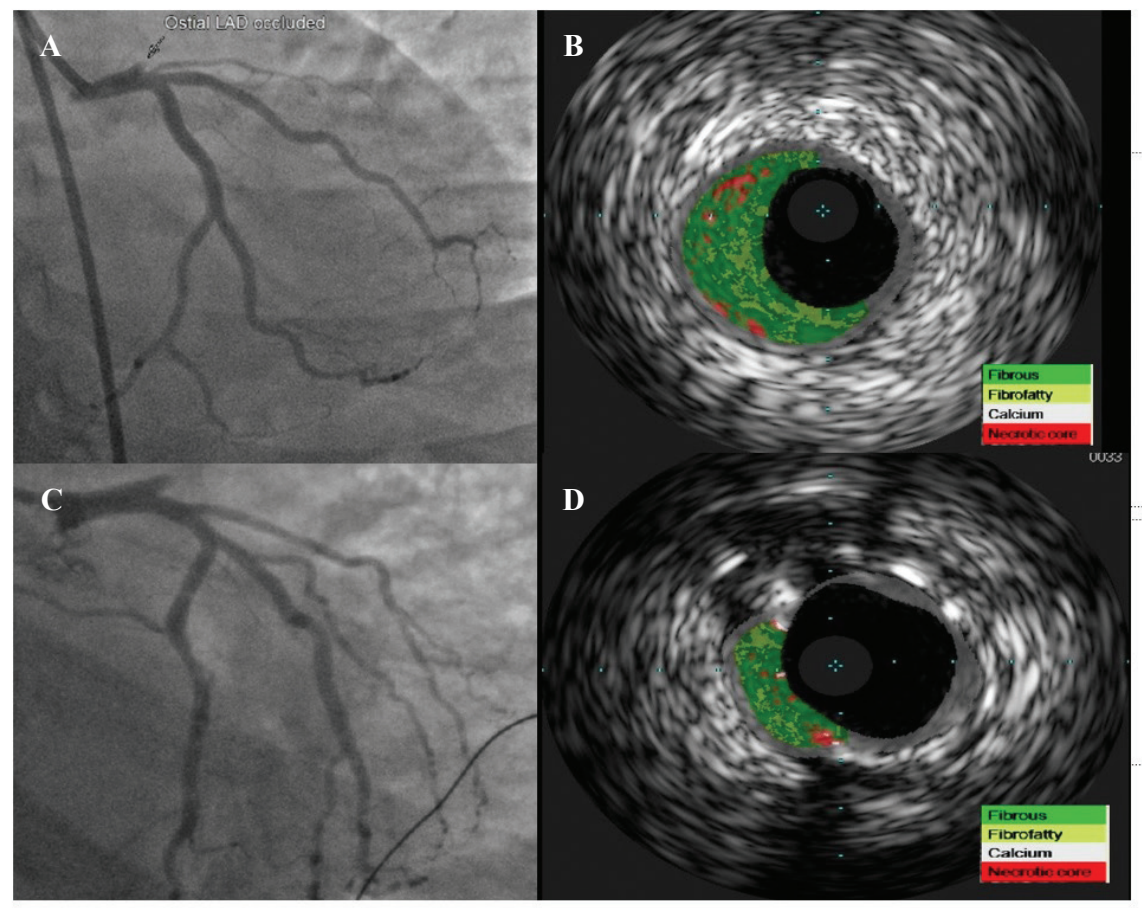

Fig. 1. (A) Coronary angiogram of Case 1 revealed acute occlusion of the ostial left anterior descending artery. (B) Virtual histology showed focal, heavily fibrous plaque burden of $59 \%$ with minimal necrotic core. (C) Coronary angiogram of Case 2 showed acute occlusion of the left anterior descending artery. (D) Subsequent virtual histology showed predominantly fibrous plaque burden of $31 \%$.

positive lupus anticoagulant. A further workup did not reveal any evidence of hyperlipidaemia, diabetes mellitus, thyroid disorder or metabolic syndrome.

The patient's hospitalisation was complicated by lower limb compartment syndrome, which was treated with fasciotomy; acute kidney injury; and critical illness neuromyopathy. Tracheostomy was performed in view of prolonged mechanical ventilation and myopathy. He is currently undergoing intensive rehabilitation in our institution.

Case 2. A 50-year-old man presented to the emergency department for worsening chest pain. Initial physical examination revealed his blood pressure as $116 / 93 \mathrm{mmHg}$; a heart rate of 120 beats per minute; and oxygen saturation $100 \%$ (on 2 litres of supplementary oxygen). Heart sounds were normal without murmurs and there were no abnormalities detected on lung auscultation. The man presented 174 days after his positive SARSCoV-2 antibody test result on 8 June 2020 . He developed a VF arrest while waiting for an ECG, where immediate resuscitation and defibrillation were carried out successfully. The ECG post-defibrillation showed ST-segment elevation in the anterior leads. The patient was subsequently intubated and transferred to the cardiac catheterisation laboratory. Coronary angiogram revealed acute thrombotic occlusion of the ostial segment of LAD. Virtual histology (via IVUS) revealed a predominantly fibrous plaque ( $31 \%$ burden) that was successfully treated through thrombectomy, followed by stent implantation (Figs. 1C and 1D). Given the thrombus burden, he was treated with glycoprotein IIb/IIIa inhibitor (eptifibatide) infusion. TTE showed mild left ventricular systolic dysfunction (left ventricular ejection fraction 45\%) with hypokinesia in the apex and anterior wall.

The patient had a high fibrinogen level of $5.7 \mathrm{~g} / \mathrm{L}$ $(1.8-4.5 \mathrm{~g} / \mathrm{L})$; elevated VWF level of $215 \%(56-160 \%)$; factor VIII level of $338 \%$; and a raised D-dimer level of $1.02 \mu \mathrm{g} / \mathrm{mL}(<0.5 \mu \mathrm{g} / \mathrm{mL})$. His cardiovascular risk factor screening panel and SARS-CoV-2 RT-PCR results were negative. Clot waveform analysis (CWA) through activated partial thromboplastin time using automated blood coagulation analyser CN-6000 (Sysmex Corp, Kobe, Japan) found a markedly elevated median maximum velocity (Min 1 ) of $8.007 \% / \mathrm{s}$ (reference range 2.86-6.78/s), elevated median maximum acceleration (Min2) of 1.341 (reference range 0.46-1.10), and a high median delta change of $68.6 \% / \mathrm{s}$ (reference range 25.21-63.09) (Fig. 2).

The patient's clinical and haemodynamic condition improved gradually. He was extubated and discharged after 12 days of hospitalisation. 


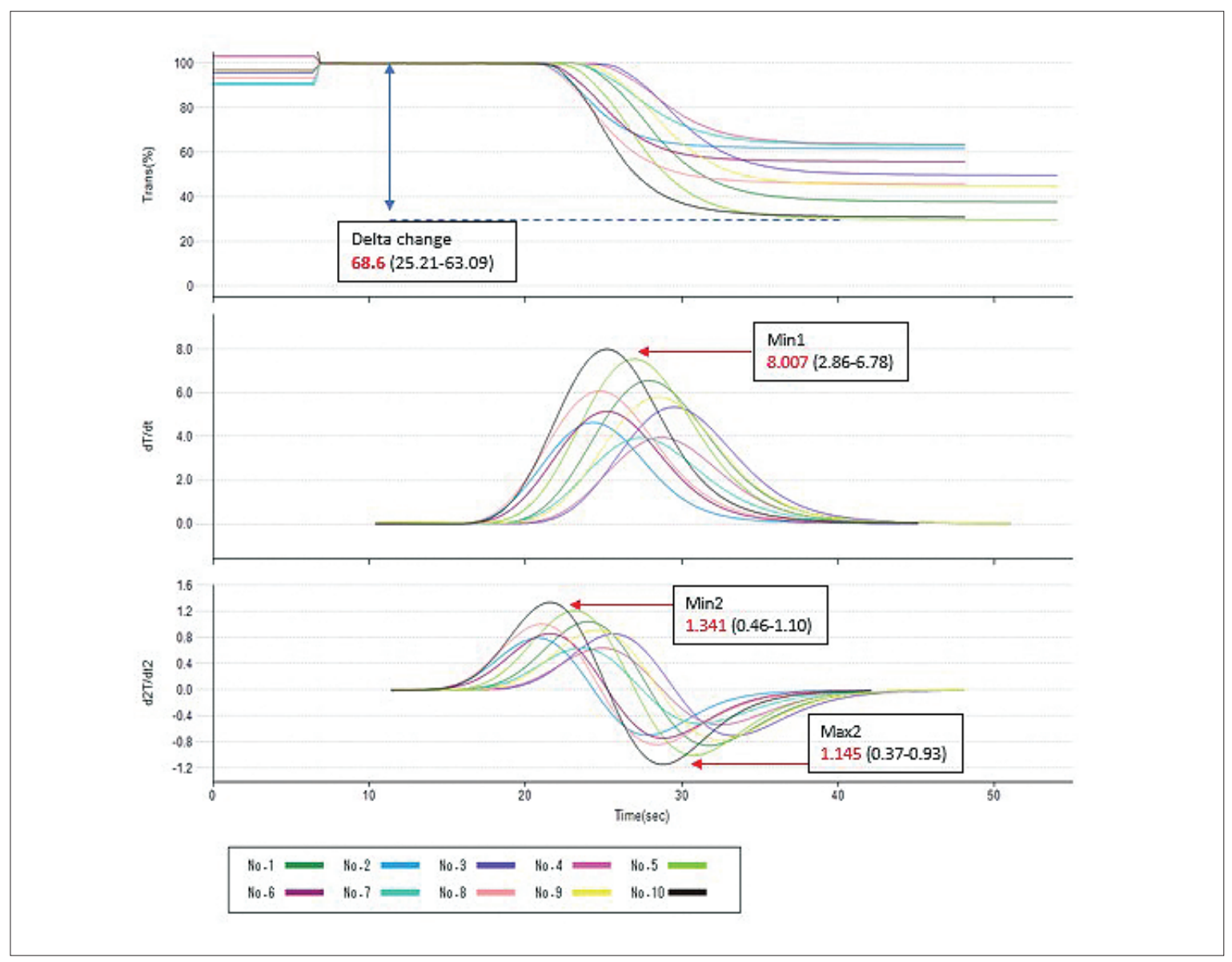

Fig. 2. Clot waveform analysis of Case 2. Patient's tracing is in black; healthy controls (for reference) in multicolour. CWA showed markedly elevated median Min1 of $8.007 \% / \mathrm{s}$, elevated median Min2 of 1.341 and high median delta change of $68.6 \% / \mathrm{s}$.

Min1: maximum velocity; Min2: maximum acceleration; Max2: maximum deceleration

Case 3. A 36-year-old man was admitted to our hospital with a persistent chest pain of 3 hours. On admission, his blood pressure and heart rate were $120 / 88 \mathrm{mmHg}$ and 88 beats per minute, respectively. Oxygen saturation was $100 \%$ on room air with normal lung auscultation. ECG showed hyperacute $\mathrm{T}$ waves in the anterior leads and his RT-PCR results for COVID-19 was negative. He presented 165 days after his positive SARS-CoV-2 antibody serology on 18 June 2020. He demonstrated a raised factor VIII level of $162 \%$ and a VWF level of $92 \%$. Coronary angiogram revealed an acute mid-LAD occlusion, for which he underwent angioplasty with successful implantation of a drug-eluting stent (Fig. 3). After percutaneous coronary intervention, the chest pain and ST-segment deviation resolved. The patient was discharged well on day 6 of his hospitalisation.

While it is known that approximately $30 \%$ of myocardial infarctions are preceded by an upper respiratory infection, in particular influenza; less is known about the thrombotic sequelae in COVID-19 during the convalescent period. ${ }^{7,8}$ We described 3 cases of COVID-19 recovered patients who presented with AMI. The remarkable characteristics of these cases included their relatively young age without preexisting cardiovascular risk factors, preceding asymptomatic SARS-CoV-2 infection, and the presence of a long latency period between initial positive SARS-CoV-2 serology and their AMI.

Thrombosis has been classically associated with Virchow's triad of blood stasis, endothelial activation, and hypercoagulable state. However, in the convalescent phase after a COVID-19 infection, studies have described a waning hypercoagulable state with possible persistence of endothelial dysfunction in patients. This has been well described in children during their recovery from COVID-19.

Multisystem inflammatory syndrome in children (MIS-C) is a newly defined post-viral myocarditis and inflammatory vasculopathy of children following COVID-19 infection. MIS-C is likely due to viral trophism of myocardial and endothelial cells by the 


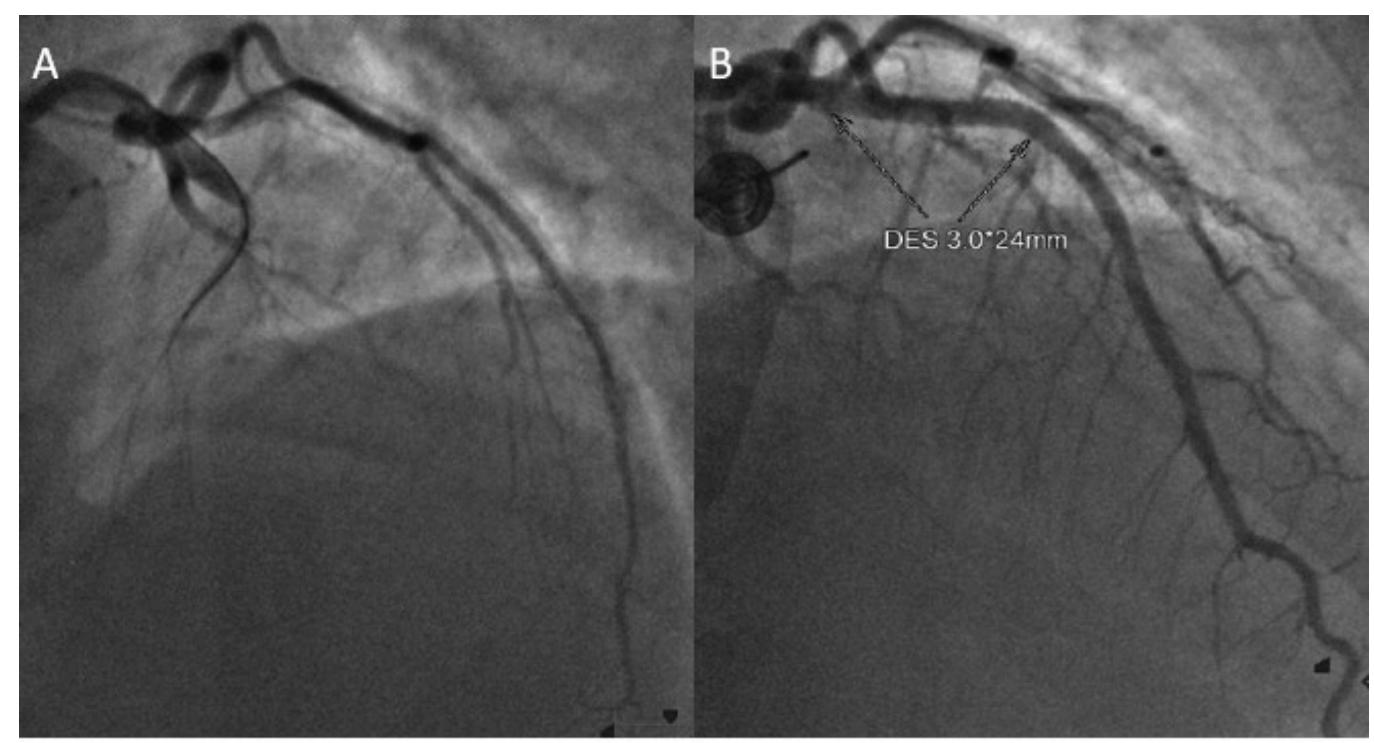

Fig. 3. (A) Coronary angiogram of Case 3 showed an occlusion of mid left anterior descending artery. (B) Re-establishment of blood flow after implantation of drug eluting stent.

coronavirus. The best evidence supporting MIS-C have been demonstrated in paediatric cases presenting with self-limited, chilblain-like acral purpuric lesion. ${ }^{9}$ The children remain otherwise asymptomatic, and interestingly, often test negative for SARS-CoV-2 in nasopharyngeal samples. Some acute viral infections are associated with transiently elevated lupus anticoagulant, but they can persist and lead to thromboembolic complications by various mechanisms, including the release of microparticles and exposure of prothrombotic phospholipids. ${ }^{10}$ Although the significance of these antibodies is not well established yet, COVID-19induced lupus anticoagulant could favour the occurrence of thromboembolic events in children populations and hence should be systematically tested for.

Virtual histology via IVUS revealed heavily fibrous plaques in the coronary arteries of the cases described. This is unusual as fibrotic lesions are usually deprived of lipid and inflammatory cells, and hence less likely to rupture and generate thrombosis. ${ }^{11}$ A fibrotic plaque consists mainly of fibrous tissue without a necrotic core or calcium. ${ }^{12,13}$ This type of plaque is mostly indolent and stable in comparison with thin cap fibroatheroma, the main culprit in acute coronary syndrome. Interestingly, in the current cases described, delayed thrombotic arterial events occurred 80-174 days from the onset of positive SARS-CoV-2 serology. Laboratory evaluation of the haemostatic profiles with raised factor VIII, VWF and D-dimer supported an ongoing vasculopathy. The CWA, a global haemostatic test that was performed on case 2, demonstrated parameters of hypercoagulability. There were increased (1) clot Min1 "thrombin burst"; (2) peak of second derivative curve; (3) clot maximum deceleration; and (4) delta change (decreased light transmission reflective of increased clot thickness) as demonstrated by Fan et al. ${ }^{14}$ In addition, autopsy series demonstrated the presence of cardiac microemboli despite the absence of viral particles in the myocardium. ${ }^{15}$

Our case series suggest that life-threatening myocardial infarction can occur unexpectedly in otherwise healthy patients with asymptomatic COVID-19 infection. Physicians should have a high index of suspicion in managing patients in the convalescent phase. Screening for and strict management of cardiovascular risk factors are of utmost importance post COVID-19. Further longitudinal studies in patients with "long COVID" should be performed to look for post-COVID-19 associated endotheliopathy and thrombotic sequelae, where there may be a role for thromboprophylaxis in high-risk groups.

\section{REFERENCES}

1. Gu SX, Tyagi T, Jain K, et al. Thrombocytopathy and endotheliopathy: crucial contributors to COVID-19 thromboinflammation. Nat Rev Cardiol 2021;18:194-209.

2. Tang N, Li D, Wang X, et al. Abnormal coagulation parameters are associated with poor prognosis in patients with novel coronavirus pneumonia. J Thromb Haemost 2020;18:844-7.

3. Lillicrap D. Disseminated intravascular coagulation in patients with 2019-nCoV pneumonia. J Thromb Haemostat 2020;18:786-7.

4. Fan BE, Umapathi T, Chua K, et al. Delayed catastrophic thrombotic events in young and asymptomatic post COVID-19 patients. J Thromb Thrombolysis 2021;51:971-7. 
5. Chen JI, Yap JCH, Hsu LY, et al. COVID-19 and Singapore: From early response to circuit breaker. Ann Acad Med Singap 2020; 49:561-72.

6. Tan THY, Toh MPHS, Vasoo S, et al. Coronarvirus disease 2019 (COVID-19): The Singapore experience. A review of the first eight months. Ann Acad Med Singap 2020;49:764-78.

7. Spodick DH, Flessas AP, Johnson MM. Association of acute respiratory symptoms with onset of acute myocardial infarction: prospective investigation of 150 consecutive patients and matched control patients. Am J Cardiol 1984;53:481-2.

8. Bainton D, Jones GR, Hole D. Influenza and ischaemic heart disease: a possible trigger for acute myocardial infarction? Int J Epidemiol 1978;7:231-9.

9. Hernandez C, Bruckner AL. Focus on "COVID Toes". JAMA Dermatol 2020;156:1003.

10. Abdel-Wahab N, Talathi S, Lopez-Olivo MA, et al. Risk of developing antiphospholipid antibodies following viral infection: a systematic review and meta-analysis. Lupus 2018; 27:572-83.

11. Bharadwaj AS, Vengrenyuk Y, Yoshimura T, et al. Multimodality intravascular imaging to evaluate sex difference in plaque morphology in stable CAD. JACC Cardiovasc Imaging 2016;9:400-7.

12. Honda S, Kataoka Y, Kanaya T, et al. Characterization of coronary atherosclerosis by intravascular imaging modalities. Cardiovasc Diagn Ther 2016;6:368-81.

13. Bentzon JF, Otsuka F, Virmani R, et al. Mechanisms of plaque formation and rupture. Circ Res 2014;114:1852-66.

14. Fan BE, Ng J, Seok SSW, et al. COVID-19 associated coagulopathy in critically ill patients: A hypercoagulable state demonstrated by parameters of haemostasis and clot waveform analysis. J Thromb Thrombolysis 2021;51:663-74.

15. Guagliumi G, Sonzogni A, Pescetelli I, et al. Microthrombi and ST-segment elevation myocardial infarction in COVID-19. Circulation 2020;142:804-9. 